\title{
Values of Default Mode Network to Alzheimer's Disease Call for Consilience of Multimodal Neuroimaging and Genetics
}

\author{
Bing $\mathrm{Hou}^{1,2}$ and Lijuan $\mathrm{Xu}^{1}$ \\ ${ }^{1}$ LIAMA Center for Computational Medicine, National Laboratory of Pattern Recognition, Institute of Automation, Chinese Academy of Sciences, Beijing, \\ 100190, People's Republic of China, and 2Beijing Institute of Radiation Medicine, Beijing, 100850, People's Republic of China \\ Review of Hedden et al.
}

\begin{abstract}
Alzheimer's disease (AD) is the most common form of dementia found among the old, but its pathogenesis is not well understood. No drug is available to stop or cure the neurodegenerative disorder; currently used treatments offer only small symptomatic benefits. However, AD research has developed to a point where scientists can look beyond treating symptoms to think about many possible interventions delaying or preventing $\mathrm{AD}$. Accordingly, it is imperative that researchers and clinicians identify the earliest possible biomarkers for the diagnosis of $\mathrm{AD}$.

During the past few years, dramatic breakthroughs have been made regarding neuroimaging of $\mathrm{AD}$. With the help of ${ }^{11}$ C-labeled Pittsburgh Compound B (PiB), positron emission tomography (PET) can reliably assess amyloid deposition, which is one of the hallmark pathologies of $\mathrm{AD}$, in the brain in vivo (Klunk et al., 2004). Consistent with neuropathological observations, PiB PET confirmed that a substantial proportion of clinically normal elders harbored amyloid deposition (Johnson et al., 2007). Although amyloid deposition occu-
\end{abstract}

Received Dec. 2, 2009; revised Jan. 4, 2010; accepted Jan. 6, 2010.

This work was supported by the National Natural Science Foundation of China (30730035). We thank Prof. Tianzi Jiang and Drs. Bing Liu and Ming Song (National Laboratory of Pattern Recognition) for their insightful comments on this article.

Correspondence should be addressed to Dr. Bing Hou, LIAMA Center for Computational Medicine, National Laboratory of Pattern Recognition, Institute of Automation, Chinese Academy of Sciences, Beijing, 100190, People's Republic of China. E-mail: hou_bing@hotmail.com.

D0I:10.1523/JNEUROSCI.6008-09.2010

Copyright $\odot$ 2010 the authors $\quad 0270-6474 / 10 / 303553-02 \$ 15.00 / 0$ pies the central position in theories of $\mathrm{AD}$ causation, the functional consequences of amyloid deposition in this population remain unknown.

As a noninvasive in vivo imaging technique, functional magnetic resonance imaging (fMRI) has been recognized as a valuable tool to identify the brain dysfunction. Typically, fMRI measures blood oxygen level-dependent (BOLD) signal correlating with task-induced changes in neural activity. In the resting state without task demand (such as visual fixation), there are spatially coherent, spontaneous fluctuations in BOLD signal among a set of brain structures comprising the socalled "default mode network" (DMN), which is believed to contribute to internal mentation including self-reflective thoughts and judgments, perception of the mental states of others, etc. (Buckner et al., 2008).

Given that some structures within DMN are among the most commonly affected regions in AD (Klunk et al., 2004), amyloid deposition might cause DMN dysfunction before dementia symptoms emerge. In a study recently published in The Journal of Neuroscience, Hedden et al. (2009) attempted to explore the functional consequences of amyloid deposition in clinically normal older adults, by combining use of PiB PET and restingstate fMRI. The clinically normal older participants were given intravenous injections of ${ }^{11} \mathrm{C}$-labeled PiB for assessment of amyloid deposition via PET imaging, and then divided into $\mathrm{PiB}$-positive and $\mathrm{PiB}$ - negative individuals. Compared with the PiB-negative individuals, functional connectivity within DMN was significantly reduced in the $\mathrm{PiB}$-positive counterparts [Hedden et al. (2009), their Fig. 2a]. If PiB level was taken as a continuous rather than dichotomous measure, there was still a significant negative correlation between $\mathrm{PiB}$ level and functional connectivity within DMN, with connectivity decreasing as PiB level increased [Hedden et al. (2009), their Fig. 2b]. The correlation with amyloid deposition seemed specific to DMN, because no significant intergroup difference in functional connectivity was found either between left and right motor regions or between left and right visual regions. These findings were soon confirmed by another study almost simultaneously published by an independent team (Sheline et al., 2009). The work by Hedden et al. (2009), together with other studies (Sheline et al., 2009; Sperling et al., 2009), consistently revealed that the older adults defined as clinically normal by standard neuropsychological tests were not homogeneous in DMN function. Thus, the "normal elders" used as controls for patients with $\mathrm{AD}$ or other types of dementia in many previous studies might be more heterogeneous in DMN function than assumed.

It is worth noting that Hedden et al. (2009) performed resting-state fMRI scanning on the participants. This experimental paradigm is of special significance for ADrelated fMRI studies, because task-related fMRI can be difficult to carry out on patients 
with $\mathrm{AD}$ or other types of dementia. Using resting-state fMRI, Hedden et al. (2009) obtained results consistent with those found in another fMRI study that used a face-name pair task (Sperling et al., 2009).

The Hedden et al. (2009) study linked DMN dysfunction to amyloid deposition found in clinically normal elders, which supports the hypothesis that disruption of functional connectivity within DMN could be an alternative phenotypic marker for early diagnosis of $\mathrm{AD}$ (Greicius et al., 2004). Obviously, it is valuable to distinguish people with $\mathrm{AD}$ from normal elders using a sensitive but noninvasive imaging technique like fMRI at a stage when canonical neuropsychological tests are hardly able to identify the affected individuals. Of course, further validation of the hypothesis requires longitudinal observation of these clinically normal older participants. In consideration of the comparatively weak correlation $(r=-0.40, p<0.02)$ between DMN dysfunction and amyloid deposition, it is so far premature to predict $\mathrm{AD}$ merely based on DMN function. Before DMN function analysis is proven clinically useful for early diagnosis of $\mathrm{AD}$, large population studies are essential to eliminate possible falsepositive and/or false-negative correlations.

Also importantly, the causality between amyloid deposition and DMN dysfunction remains to be determined. On the one hand, the observed DMN dysfunction might be one of the functional consequences of amyloid deposition. On the other hand, DMN dysfunction might be one of principal causes of amyloid deposition, given that high levels of neural activity can result in amyloid accumulation in vivo (Cirrito et al., 2005) and that nondemented elders with amyloid burden fail to deactivate DMN during memory tasks (Sperling et al., 2009). Perhaps most likely, amyloid deposition and DMN dysfunction might aggravate each other in the course of AD. The recently developed high-field fMRI, together with other invasive techniques, will offer a chance to clarify this question in animals.
This study indicated that amyloid deposition could not fully explain alterations in functional connectivity within DMN. Are there other contributors? Hedden et al. (2009) did not answer this question. We speculate that the gene polymorphisms ubiquitously present among the population may be the primary candidates. It has recently been shown that young healthy subjects carrying ApoE $\varepsilon 4$ allele, the best-established genetic risk factor for $\mathrm{AD}$, exhibited altered $\mathrm{DMN}$ function decades before any clinical symptom could be identified (Filippini et al., 2009), suggesting polymorphisms in ApoE can modulate functional connectivity within DMN. Undoubtedly, neuroimaging combined with genetics will open new avenues to understanding the genetic basis for variations of DMN function. Given the known functions of DMN (Buckner et al., 2008), it is reasonable to predict that genetic variation in other cognition-related proteins may also contribute to variations of DMN function in clinically normal subjects. Among the likely candidates is catechol-O-methyltransferase (COMT), a key enzyme responsible for most of the dopamine degradation in the prefrontal cortex (PFC), a hub of DMN. Because COMT variants have different catalytic abilities, we expect to see that COMT gene polymorphisms modulate functional connectivity within DMN.

In conclusion, Hedden et al. (2009) reported that disruption of functional connectivity within DMN was associated with amyloid disposition present in a substantial proportion of clinically normal elders. Their findings favor DMN dysfunction being an alternative marker for early diagnosis of $\mathrm{AD}$. We speculate gene polymorphisms may also contribute to the variations of DMN function. To comprehensively understand the values of DMN to $\mathrm{AD}$, it will be necessary to combine multimodal neuroimaging including $\mathrm{PiB}$ PET and restingstate fMRI, as well as genetic approaches.

\section{References}

Buckner RL, Andrews-Hanna JR, Schacter DL (2008) The brain's default network: anatomy, function, and relevance to disease. Ann N Y Acad Sci 1124:1-38.

Cirrito JR, Yamada KA, Finn MB, Sloviter RS, Bales KR, May PC, Schoepp DD, Paul SM, Mennerick S, Holtzman DM (2005) Synaptic activity regulates interstitial fluid amyloidbeta levels in vivo. Neuron 48:913-922.

Filippini N, MacIntosh BJ, Hough MG, Goodwin GM, Frisoni GB, Smith SM, Matthews PM Beckmann CF, Mackay CE (2009) Distinct patterns of brain activity in young carriers of the APOE-epsilon4 allele. Proc Natl Acad Sci U S A 106:7209-7214.

Greicius MD, Srivastava G, Reiss AL, Menon V (2004) Default-mode network activity distinguishes Alzheimer's disease from healthy aging: evidence from functional MRI. Proc Natl Acad Sci U S A 101:4637-4642.

Hedden T, Van Dijk KR, Becker JA, Mehta A, Sperling RA, Johnson KA, Buckner RL (2009) Disruption of functional connectivity in clinically normal older adults harboring amyloid burden. J Neurosci 29:12686-12694.

Johnson KA, Gregas M, Becker JA, Kinnecom C, Salat DH, Moran EK, Smith EE, Rosand J, Rentz DM, Klunk WE, Mathis CA, Price JC, Dekosky ST, Fischman AJ, Greenberg SM (2007) Imaging of amyloid burden and distribution in cerebral amyloid angiopathy. Ann Neurol 62:229-234.

Klunk WE, Engler H, Nordberg A, Wang Y, Blomqvist G, Holt DP, Bergström $M$, Savitcheva I, Huang GF, Estrada S, Ausén B, Debnath ML, Barletta J, Price JC, Sandell J, Lopresti BJ, Wall A, Koivisto P, Antoni G, Mathis CA, et al. (2004) Imaging brain amyloid in Alzheimer's disease with Pittsburgh Compound-B. Ann Neurol 55:306-319.

Sheline YI, Raichle ME, Snyder AZ, Morris JC, Head D, Wang S, Mintun MA (2009) Amyloid plaques disrupt resting state default mode network connectivity in cognitively normal elderly. Biol Psychiatry. Advance online publication. Retrieved Feb. 25, 2010. doi:10.1016/j.biopsych. 2009.08.024

Sperling RA, Laviolette PS, O’Keefe K, O’Brien J, Rentz DM, Pihlajamaki M, Marshall G, Hyman BT, Selkoe DJ, Hedden T, Buckner RL, Becker JA, Johnson KA (2009) Amyloid deposition is associated with impaired default network function in older persons without dementia. Neuron 63:178-188. 